\title{
Toothache as a presenting symptom of metastatic renal cell cancer
}

\author{
Abhirami Hallock, MD, BSc; George Rodrigues, MD, FRCPC, MSc
}

\begin{abstract}
A 53-year-old man with a 14-year history of renal cell carcinoma (RCC) presented with a 2-month history of right-sided upper jaw pain and severe bleeding during tooth extraction. Pathology review of a lower maxillary sinus/upper gingival mass revealed metastatic RCC. The presentation, differential diagnosis and literature review of this uncommon presentation of metastatic kidney cancer are discussed in this report.
\end{abstract}

Can Urol Assoc J 2009;3(5):E42-E44

\section{Case report}

In 1994, this patient, at 39 years old, was diagnosed with renal cancer, with an initial presentation of epigastric and subcostal pain. An 8-cm left kidney mass was found on imaging and he subsequently underwent a left radical nephrectomy. Pathologic evaluation showed a well-encapsulated clear cell with no penetration into the vasculature or perinephric fat consistent with stage II (T2NOM0) renal cell carcinoma (RCC). A solitary metastatic lesion to the cerebellum was found after he presented with ataxia and headaches in 1997. He underwent a total resection followed by postoperative cranial radiation and was discharged from the cancer centre. He underwent an uneventful follow-up after 5 years.

He remained symptom-free until mid-2007, when he started experiencing upper jaw pain and underwent dental evaluation. Preoperative dental x-rays revealed extensive bone loss at the right upper second molar and erosion into the buccal and palatal bone with extension into the first molar. Discomfort was believed to be secondary to periodontal disease and tooth extraction was recommended. A mass in the gingiva was noted and both the tooth and biopsy tissue were sent for pathological assessment, which was ultimately consistent with RCC.

His staging workup revealed a large expansile mass in the maxilla measuring $2.5 \times 2.1 \times 2.8 \mathrm{~cm}$ with extension to the maxillary sinus, a large subcarinal node measuring $5 \mathrm{~cm}$ and multiple small pulmonary nodules (Fig. 1). No radiological evidence of contralateral kidney or adrenal disease was found. Bone scan showed asymptomatic lesions in the right maxilla and the T9 vertebra.

On history, he denied hematuria, epistaxis, parenthesis, shortness of breath, constitutional symptoms or any change in performance status. His physical examination was unremarkable, with no obvious facial deformity or palpable mass over the alveolar ridge. Laboratory work revealed mild anemia with a hemoglobin of $134 \mathrm{~g} / \mathrm{L}$, lactate dehydrogenase within normal limits at $130 \mathrm{U} / \mathrm{L}$ and creatinine clearance at $121 \mathrm{~mL} / \mathrm{min}$.

The patient underwent radiation treatment to the maxilla, at a dose of 50 Gy in 20 fractions over 4 weeks to achieve local control of the disease. He was also evaluated by a medical oncologist for therapy with a targeted agent and began sunitinib (an oral vascular endothelial growth factor receptor kinase blocker) after completion of radiotherapy. Posttreatment, he developed severe mucositis requiring antibiotics. Repeat imaging of the head and thorax showed stable disease in the maxilla and regression of the lung nodules 5 months after completion of treatment.

\section{Discussion}

Renal cell carcinoma is the seventh most common cancer in Canada, mostly affecting males between their fifth and sixth decades. Renal cancers are more commonly incidental findings with the increased use of computed tomography scan technology. They have been referred to as the "internist tumour" ${ }^{1}$ because presenting symptoms vary widely from the classic triad of hematuria, flank pain and palpable mass. Most common histologies for these cancers are clear cell (80\%), papillary, chromophil and chromophobic tumours (Box 1). The metastatic potential of these histologies can vary from very aggressive collecting-duct tumours to indolent chromophobic and oncocytic lesions. Stage is an important prognostic factor and $25 \%$ of patients have locally advanced or metastatic disease at presentation., 1,2

The ability of renal carcinomas to metastasize after prolonged periods and their highly variable clinical course are well-described in the literature. Metastatic dissemination 


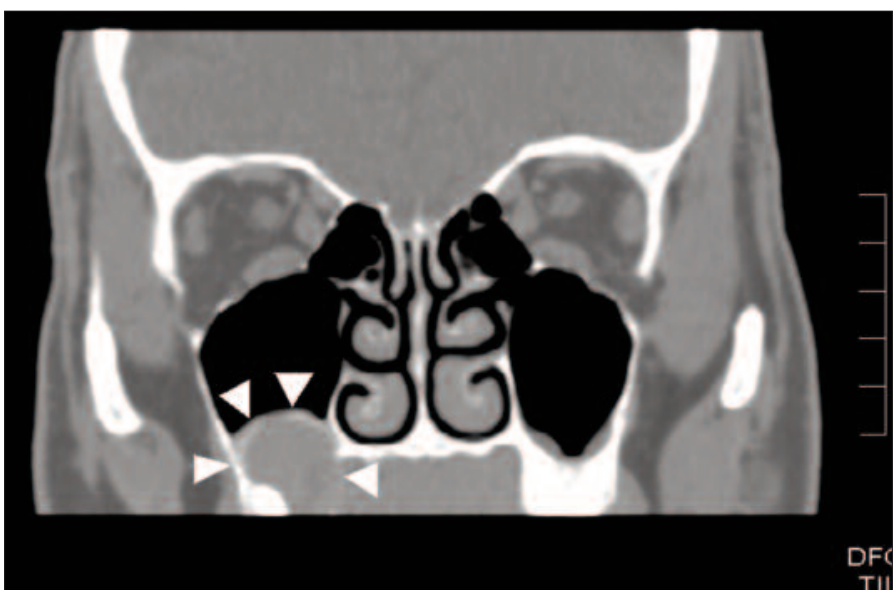

Fig. 1. Coronal section showing mass involving the maxilla with extension into maxillary sinus.

can be widespread and most common to lung $(75 \%)$, regional lymph nodes $(66 \%)$, bone $(40 \%)$ and liver $(40 \%)$. Pathogenesis of metastatic renal cell cancers of the head and neck region has been reviewed in several reports. It is hypothesized that the valveless vertebral venous plexus allows the tumour emboli to bypass the pulmonary filter and enter the cranial areas. Bony metastases depend on hematogenous spread, with most bony lesions found in structures with large red bone marrow content like the vertebrae, ribs and femur. The mandible is more commonly involved than the maxilla because of its greater red marrow content. Only $10 \%$ of lesions in the jaw are in the maxilla.

Forty percent of patients are treated for localized tumour relapse and a third of patients develop incurable distant metastases. Five-year survival for patients with metastases is poor, at less than $10 \%$, with a median survival rate of approximately 13 months. ${ }^{1}$ Patients with metastatic oral tumours tend to have lesions at other sites and have poor prognosis. Metastectomy can be performed for solitary metastases and has an overall 5 -year survival rate of $35 \%$ to $50 \%$. Median survival for patients with jaw metastases is poor, at 6 months (range 1 to 60 months). Local treatment of jaw metastases frequently involves radiotherapy and/or surgery for local control of disease, pain relief and prevention of loss of function.

Box 1. Differential diagnosis for clear cell histology

- Mucoepidermoid carcinoma

- Acinic cell carcinoma

- Odontogenic carcinoma with clear cells

- Calcifying odontogenic tumour

- Thyroid

- Breast

- Colon
There are only sporadic cases recorded in the English medical literature of renal metastases to the maxilla/ mandible. ${ }^{3-8}$ Interestingly, there is 1 similar case reported in the literature ${ }^{3}$ of a patient with a prior cerebral lesion before metastases to maxilla. The timelines in that report were shorter, with cerebral metastasis diagnosed 7 years after diagnosis and metastases to maxilla 1 year later, with no other sites of metastases. The management of this patient consisted of debulking surgery and embolization. Death occurred 2.5 years after embolization.

\section{Conclusion}

We present a case of a patient who presented with a second metastatic lesion 14 years after initial diagnosis. Low index of suspicion or failure to obtain tissue pathology would have delayed diagnosis and perhaps had a negative impact on quality of life and survival. Early discovery of his jaw metastases and asymptomatic lung metastases allowed him to undergo aggressive radiotherapy and targeted medical therapy.

Dental symptoms can be the first sign of relapse; we propose that physicians and dentists keep a high level of index for metastatic disease, even after prolonged disease-free intervals in patients with a history of cancer and RCCs in particular (Box 2, Box 3).

From the Department of Radiation Oncology, London Regional Cancer Program, London, ON

This paper has been peer-reviewed.

Competing interests: None declared.

Box 2. Differential diagnosis for lesions in the mandible

- Osteomyelitis

- Pyogenic granuloma

- Metastatic lesions

- Mandibular cyst

- Neoplasm: benign or malignant

- Systemic disease with bone involvement

Box 3. Possible symptoms from jaw metastases

- Asymptomatic

- Pain

- Paraesthesia (numb chin syndrome)

- Loosening of tooth

- Local swelling

- Pathologic fractures 


\section{References}

1. Reaume MN, Canil CM. Advanced renal cell carcinoma: hitting the target with novel systemic therapies. Oncology Exchange 2007;6:10-3.

2. Cohen H, McGovern F. Renal cell carcinoma. N Engl J Med 2005;353:2477-88.

3. Ord RA, Malins T, Ward-Booth PR. Vascular metastatic renal carcinoma of the maxilla: Report of two cases. Int J Oral Maxillofac Surg 1990;19:106-9.

4. Akdas A. Kirkali Z, Ruacan S, et al. Unusual metastases of renal cell carcinoma. Urol Int 1987;42:316-7.

5. Lee G, Sharma SD, Bullock KN. An unusual case of renal cell carcinoma with two rare metastases. Scand J Urol Nephrol 1998;32:239-40.
6. Terada N, Hiruma K, Suzuki M, et al. Metastasis of renal cell cancer to the ethmoid sinus. Acta Otolaryngol Suppl 1998:537:82-6.

7. Sastre J, Naval L, Munoz M, et al. Metastatic renal cell carcinoma to the mandible. Otolaryngol Head Neck Surg 2005;132:663-4.

8. Van der Waal RI, Buter J, van der Waal I. Oral metastases: report of 24 cases. Br J Oral Maxillofac Surg 2003;41:3-6.

Correspondence: Dr. George Rodrigues, London Regional Cancer Program, London Health Sciences Centre, 800 Commissioners Road East, P0 Box 5010, London, ON N6A 5W9; fax 519-6858736; george.rodrigues@|hsc.on.ca 